Bull. Austral. Math. Soc.

Vol. 48 (1993) [379-383]

\title{
REMARKS CONCERNING THE 2-HILBERT CLASS FIELD OF IMAGINARY QUADRATIC NUMBER FIELDS
}

\author{
Elliot Benjamin
}

\begin{abstract}
Let $k$ be an imaginary quadratic number field and let $k_{1}$ be the 2-Hilbert class field of $k$. If $C_{k, 2}$, the 2-Sylow subgroup of the ideal class group of $k$, is elementary and $\left|C_{k, 2}\right| \geqslant 8$, we show that $C_{k_{1}, 2}$ is not cyclic. If $C_{k, 2}$ is isomorphic to $Z / 2 Z \times Z / 4 Z$ and $C_{k_{1}, 2}$ is elementary we show that $k$ has finite 2-class field tower of length at most 2 .
\end{abstract}

\section{INTRODUCTION}

Let $k$ be a number field and let $C_{k, 2}$ denote its 2-class group, that is the 2-Sylow subgroup of the ideal class group, $C_{k}$, (in the wider sense) of $k$. We let $k_{1}$ denote the 2-Hilbert class field of $k$; that is the maximal unramified (including the infinite primes) Abelian field extension of $k$ which has degree a power of 2 . It is well known that if $C_{k, 2}$ is elementary of rank 2 , that is isomorphic to $Z / 2 Z \times Z / 2 Z$, which we shall denote by $(2,2)$, then $C_{k_{1}, 2}$ is cyclic (see Kisilevsky [6], Gorenstein [4], Taussky [8]). However, in the case where $k$ is an imaginary quadratic number field and $C_{k, 2}$ is elementary of rank greater than 2 , we show that $C_{k_{1}, 2}$ is not cyclic.

We next examine the case where $k$ is an imaginary quadratic number field, $C_{k, 2}$ is isomorphic to $Z / 2 Z \times Z / 4 Z$ (denoted by $(2,4)$ ), and $C_{k_{1}, 2}$ is elementary. From our earlier paper (see Snyder and Benjamin [7]), and the group tables in Hall and Senior [5] we are able to show that $C_{k_{1}, 2}$ does not have rank 3 , which implies that $k$ has finite 2-class field tower of length at most 2 (see Blackburn [1, 2]). In the process of examining various groups $G$ of order 64 with $G / G^{\prime} \cong(2,4)$ we have uncovered some errors in Hall and Senior [5] which we describe.

\section{Results When $C_{k, 2}$ IS ELEMENTARY}

We begin by stating a result that characterises the Galois group of the second Hilbert 2-class field, $k_{2}$, over $k$ for $k$ a number field and $C_{k, 2} \cong(2,2)$. We let $Q_{m}$, $D_{m}, S_{m}$ be the quaternion, dihedral, and semidihedral groups of order $2^{m}$. It is well known that (see Gorenstein [4]):

Received 24 November 1992

Copyright Clearance Centre, Inc. Serial-fee code: 0004-9729/93 \$A2.00+0.00. 
LEMMA 1. If $G$ is a 2 -group of order $2^{m}, m>2$, such that $G / G^{\prime} \cong(2,2)$, then $G$ is isomorphic to $D_{m}, Q_{m}$ or $S_{m}$. In particular $G^{\prime}$ is cyclic.

We now define $G=\mathrm{Gal}\left(k_{2} / k\right)$. Then from class field theory we know that $G^{\prime}=$ $\operatorname{Gal}\left(k_{2} / k_{1}\right) \cong C_{k_{1}, 2}$ and $G / G^{\prime} \cong \mathrm{Gal}\left(k_{1} / k\right) \cong C_{k, 2}$ where $G^{\prime}$ is the commutator subgroup of $G$ (see Gorenstein [4]). We can therefore conclude that if $k$ is a number field with $C_{k, 2} \cong(2,2)$ then $C_{k_{1}, 2}$ is cyclic.

At this point, assuming $k$ to be an imaginary quadratic number field, we are able to formulate an interesting contrast when $C_{k, 2}$ is elementary of rank greater than 2 . We let $L$ be an unramified quadratic extension of $k$ with $H=\operatorname{Gal}\left(k_{2} / L\right)$ and let $j$ be the homomorphism from $C_{k} \rightarrow C_{L}$ induced by the extension of ideals from $k$ to $L$. The ideal classes in the kernel of $j$, that is those ideal classes which become principal in $L$, are said to "capitulate" in $L$. It is well known that if $k$ is an imaginary quadratic number field then $|\operatorname{ker} j|=2$ if $N_{L / k}\left(E_{L}\right)=\{1,-1\}$ and $|\operatorname{ker} j|=4$ if $N_{L / k}\left(E_{L}\right)=\{1\}$ where $E_{L}$ denotes the group of units in $L$ and $N_{L / k}\left(E_{L}\right)$ denotes the norm from $L$ to $k$ of $E_{L}$. We know that the order of any ideal class in ker $j$ divides $[L: k]=2$ (see for example Furtwangler [3]) and ker $j$ is therefore contained in $C_{k, 2}$. The Artin map $\phi$ induces the following commutative diagram:

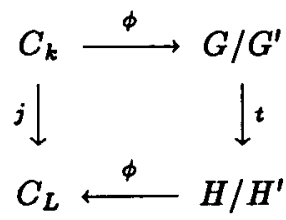

the rows are isomorphisms and $t: G / G^{\prime} \rightarrow H / H^{\prime}$ is the group tranfer map (Verlagerung) which has the following simple characterisation when $H$ is of index 2 in $G$. Let $G=H \cup z H$; then $t\left(h G^{\prime}\right)=h z h z^{-1} H^{\prime}$ and $t\left(z h G^{\prime}\right)=(z h)^{2} H^{\prime}$. Thus $\operatorname{ker} j$ is determined by ker $t$. We are now able to prove the following theorem.

THEOREM 1. Let $k$ be an imaginary quadratic number field with $C_{k, 2}$ elementary and $\left|C_{k, 2}\right| \geqslant 8$. Then $C_{k_{1}, 2}$ is not cyclic.

Proof: Let $G=\operatorname{Gal}\left(k_{2} / k\right)$; therefore $G^{\prime}=\operatorname{Gal}\left(k_{1} / k\right) \cong C_{k_{1}, 2}$ and $G / G^{\prime}=$ $\mathrm{Gal}\left(k_{1} / k\right) \cong C_{k, 2}$. Assume $G^{\prime}$ is cyclic; therefore there exists $x, y \in G$ such that $G^{\prime}=\langle\alpha\rangle$ with $x y x^{-1} y^{-1}=\alpha$. Since $G$ has rank $\geqslant 3$ (by the Burnside Basis Theorem) there exists a maximal subgroup $H$ containing $x$ and $y$, and thus $H^{\prime}=G^{\prime}=\langle\alpha\rangle$. Since $G$ has rank $m \geqslant 3$ we let $G / G^{\prime}=\left\langle\bar{\alpha}_{1}, \bar{\alpha}_{2}, \ldots, \bar{\alpha}_{m}\right\rangle$ and examine the two cases for the transfer map $t: G . G^{\prime} \rightarrow H / H^{\prime}:$

CASE 1. If $\alpha_{i} \notin H, t\left(\bar{\alpha}_{i}\right)=\alpha_{i}^{2}(\alpha)$.

CASE 2. If $\alpha_{i} \in H, t\left(\bar{\alpha}_{i}\right)=\alpha_{i} \alpha_{j} \alpha_{i} \alpha_{j}^{-1}\langle\alpha\rangle, \alpha_{j} \notin H$. 
Since $\alpha_{i}^{2} \in\langle\alpha\rangle$ then in Case $1 t\left(\bar{\alpha}_{i}\right)=I\langle\alpha\rangle$. Since $\alpha_{i} \alpha_{j} \alpha_{i} \alpha_{j}^{-1}=\alpha_{i}^{2}\left(\alpha_{i}^{-1} \alpha_{j} \alpha_{i} \alpha_{j}^{-1}\right) \in$ $\langle\alpha\rangle$ then in Case 2 we also have $t\left(\bar{\alpha}_{i}\right)=I\langle\alpha\rangle$. Thus the kernel of $t$ is $G / G^{\prime}$. From the commutative diagram above we conclude that all ideals in $C_{k, 2}$ capitulate in the corresponding unramified quadratic extension to $H$. However $\left|C_{k, 2}\right| \geqslant 8$ and since $k$ is an imaginary quadratic number field at most 4 ideals in $C_{k, 2}$ can capitulate. Consequently we have a contradiction and therefore $C_{k_{1}, 2}$ is not cyclic.

\section{RESULTS WHEN $C_{k, 2} \cong(2,4)$}

We next examine the case where $k$ is an imaginary quadratic number field, $C_{k, 2} \cong$ $(2,4)$, and $C_{k_{1}, 2}$ is elementary. Let $G=\mathrm{Gal}\left(k_{2} / k\right)$; since $G / G^{\prime} \cong C_{k, 2} \cong(2,4)$, $G / G^{\prime}=\langle\bar{a}, \bar{b}\rangle$ for some $a, b \in G$ such that $\bar{a}^{2}=I=\bar{b}^{4}$. We note that $G$ has three maximal subgroups given by $H_{1}=\left\langle b, G^{\prime}\right\rangle, H_{2}=\left\langle a b, G^{\prime}\right\rangle, H_{3}=\left\langle a, b^{2}, G^{\prime}\right\rangle$. Let $K_{i}$ be the subfield of $k_{2}$ fixed by $H_{i}, i=1,2,3$. Then $K_{1}, K_{2}, K_{3}$ comprise all of the unramified quadratic extensions of $k$. We let $j_{i}: C_{k} \rightarrow C_{K_{i}}$ be the homomorphism induced by extension of ideals from $k$ to $K_{i}$. We use the following result implicit in our earlier work (see Snyder and Benjamin [7]).

LEMMA 2. Let $k$ be an imaginary quadratic number field with $C_{k, 2} \cong\left(2,2^{m}\right)$. If $C_{k_{1}, 2}$ is not cyclic, then $\left|\operatorname{ker} j_{1}\right|=2$ or $\left|\operatorname{ker} j_{2}\right|=2$.

We now define the 2-class field tower of $k$ as the sequence $k \leqslant k_{1} \leqslant k_{2} \ldots k_{i} \leqslant$ $k_{i+1} \ldots$ where $k_{i+1}$ is the 2-Hilbert class field of $k_{i}$. If $k_{i}=k_{i+1}$ and $i$ is minimal we say that $k$ has finite 2-class field tower of length $i$; otherwise $k$ has infinite 2-class field tower. We now state and prove the following theorem.

THEOREM 2. Let $k$ be an imaginary quadratic number field with $C_{k, 2} \cong(2,4)$ and $C_{k_{1}, 2}$ elementary. Then $k$ has finite 2-class field tower of length at most 2 .

Proof: By Blackburn [1] we know that if $G / G^{\prime} \cong(2,4)$ and $k$ has 2-class field tower of length greater than 2 , then $G^{\prime}$ must have rank $\leqslant 3$. By Blackburn [2] if $G$ has rank 2 and the rank of $G^{\prime}$ is less than or equal to 2 then $G^{\prime}$ is abelian. Therefore since $C_{k, 2} \cong(2,4), C_{k_{1}, 2}$ has rank equal to 3 . By Hall and Senior [5] there are two non-isomorphic groups $G$ with $G / G \cong(2,4)$ and $G^{\prime} \cong(2,2,2)((2,2,2)$ denotes $Z / 2 Z \times Z / 2 Z \times Z / 2 Z)$. These groups can be described as follows:

$$
\begin{gathered}
G_{1}=\langle q, s\rangle: x^{2}=y^{2}=z^{2}=1, z r z r=x, y s y s^{-1}=x, q r q r=y, \\
z s z s^{-1}=y, q s q s^{-1}=z, q^{2}=1, r^{2}=1 \\
G_{2}=\langle q, s\rangle: x^{2}=y^{2}=z^{2}=1, z r z r=x, y s y s^{-1}=x, q r q^{-1} r=y, \\
z s z s^{-1}=y, q s q^{-1} s^{-1}=z, q^{2}=x, r^{2}=1, s^{2}=r .
\end{gathered}
$$

In each case $G / G^{\prime}=\langle\bar{q}, \bar{s}\rangle, \bar{q}^{2}=1, \bar{s}^{4}=1, G^{\prime}=\langle x, y, z\rangle$ and the three maximal subgroups are $H_{1}=\left\langle s, G^{\prime}\right\rangle, H_{2}=\left\langle q s, G^{\prime}\right\rangle, H_{3}=\left\langle q, s^{2}, G^{\prime}\right\rangle$. We note that $H_{i}^{\prime}=$ 
$\langle x, y\rangle$ for $i=1,2,3$ and for each group $G$ we find the kernels of the transfer maps $t_{i}: G / G^{\prime} \rightarrow H_{i} / H_{i}^{\prime}$ are as follows: $\operatorname{ker} t_{1}=\left\langle\bar{q}, \bar{s}^{2}\right\rangle$, $\operatorname{ker} t_{2}=\left\langle\bar{q}, \bar{s}^{2}\right\rangle$, ker $t_{3}=\left\langle\bar{s}^{2}\right\rangle$. We observe that $t_{1}$ and $t_{2}$ have 4 ideals that capitulate, and $t_{3}$ has 2 ideals that capitulate. From Lemma 2 we see that this capitulation cannot occur for $k$ imaginary and therefore if $\left|C_{k_{1}, 2}\right|=8, C_{k_{1}, 2}$ is not elementary. We conclude that $k$ has 2-class field tower of length at most 2 .

In the process of attempting to refine our earlier results for $k$ imaginary, $C_{k, 2} \cong$ $(2,4)$ and $C_{k_{1}, 2}$ cyclic, we observed three groups in Hall and Senior [5] that are in error.

These groups are listed as groups $64 / 140,64 / 141$ and $64 / 143$ in Hall and Senior, and can be described as follows:

GROUP 64/140. $x^{2}=1, y^{2}=x, z^{2}=y^{-1}, q^{2}=z^{-1}, y r y^{-1} r^{-1}=x, z r z^{1} r^{-1}=y$, $q r q^{-1} r^{-1}=z, r^{2}=w, w^{2}=1$.

GROUP 64/141. $x^{2}=1, y^{2}=x, z^{2}=y^{-1}, q^{2}=z^{3}, r^{2}=w, y r y^{-1} r^{-1}=x$, $z r z^{-1} r^{-1}=y, q r q^{-1} r^{-1}=z, w^{2}=1$.

Group 64/143. $x^{2}=1, y^{2}=x, z^{2}=y^{-1}, x=w^{2}, q^{2}=z^{-1}, r^{2}=w, y r y^{-1} r^{-1}=$ $x, z r z^{-1} r^{-1}=y, q r q^{-1} r^{-1}=z$.

We note that in all three groups $G_{i}, i=1,2,3$, we have $G_{i}=\langle q, r\rangle, G_{i}^{\prime}=\langle z\rangle$, $G_{i} / G_{i}^{\prime}=\langle\bar{q}, \bar{r}\rangle$, and $Z_{i}=\langle x, w\rangle$ where $Z_{i}$ is the centre of $G_{i}$. We also note that $H_{3}$, the non-cyclic maximal subgroup as a factor group of $G^{\prime}$, is always abelian since in all three groups $H_{3}=\left\langle q, r^{2}, z\right\rangle=\langle q, w\rangle$. However, in all three groups $G_{i}$ there is a problem with the transfer map $t_{i}: G_{i} / G_{i}^{\prime} \rightarrow H_{3} / H_{3}^{\prime}$. The transfer map $t_{i}$ can be described for all three groups $G_{i}$ in the following way: $t_{i}(\bar{I})=t_{i}(\bar{z})=z r z r^{-1}=$ $y r z z r^{-1}=y r y^{-1} r^{-1}=x$. Thus the transfer map takes the identity element to an element that is not the identity, which of course cannot happen.

\section{REFERENCES}

[1] N. Blackburn, 'On prime-power groups in which the derived group has two generators', Proc. Camb. Phil. Soc. 53 (1957), 19-27.

[2] N. Blackburn, 'On prime-power groups with two generators', Proc. Camb. Phil. Soc. 54 (1958), 327-337.

[3] Ph,. Furtwängler, 'Über die Klassenzahl Abelscher Zahlkörper', Crelle 134 (1908), 91-94.

[4] D. Gorenstein, Finite groups (Harper and Row, New York, 1968).

[5] M. Hall and J.K. Senior, The groups of order $2^{n}(n \leqslant 6)$ (Macmillan, New York, 1964).

[6] H. Kisilevsky, 'Number fields with class number congruent to $4 \mathrm{mod} 8$ and Hilbert's Theorem 94', J. Number Theory 8 (1976), 271-279.

[7] C. Snyder and E. Benjamin, 'Number fields with 2-class group isomorphic to $\left(2,2^{m}\right)$ ', submitted for publication (1992). 
[5]

[8] O. Taussky, 'A remark on the class field tower', J. London Math. Soc. 12 (1937), 82-85.

Mathematics Department

Unity College

Unity ME 04988-9502

United States of America 